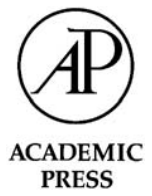
PRESS
Available online at www.sciencedirect.com

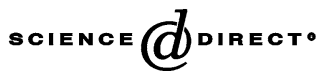

Journal of Economic Theory 122 (2005) 132-139
JOURNAL OF

Economic

Theory

http://www.elsevier.com/locate/jet

\title{
On the nonexistence of universal information structures
}

\author{
Martin Meier
}

CORE, Université Catholique de Louvain, 34, Voie du Roman Pays, B-1348 Louvain-la-Neuve, Belgium

Received 15 May 2002; final version received 29 July 2003

\begin{abstract}
This paper extends the nonexistence result of Heifetz and Samet (Games Econ. Behav. 22 (1998) 260-273). They have shown that there exists no universal knowledge space to which every knowledge space can be mapped in a knowledge-preserving manner. We show that an analogous nonexistence result holds in the more general context of information structures. These structures can be viewed as generalizations of knowledge spaces that describe nonprobabilistic beliefs.
\end{abstract}

(C) 2003 Elsevier Inc. All rights reserved.

JEL classification: $\mathrm{C} 72 ; \mathrm{D} 80 ; \mathrm{D} 82$

Keywords: Information structures; Kripke structures; Incomplete information; Interactive uncertainty

\section{Introduction}

Type spaces as introduced by Harsanyi [8] and knowledge spaces as introduced by Aumann [1] are the two prevalent models used to describe interactive uncertainty in game theory and economics. The knowledge spaces, respectively Harsanyi type spaces, used in applications are usually finite. Regardless, they typically do not contain enough states so as to represent all the potential states of mind that the players could possibly have about the interaction at hand. This poses the question as to whether the missing states prevent a correct analysis of the problem.

Mertens and Zamir [15] showed that this problem does not arise for Harsanyi type spaces. They showed that, under suitable topological assumptions on the type

E-mail address: meier@core.ucl.ac.be. 
spaces, there is a "largest" Harsanyi type space which "contains" all possible states of the world. That is, they showed the existence of a universal Harsanyi type space to which every Harsanyi type space (on the same space of states of nature) can be mapped by a morphism. A morphism is a map that preserves the state of nature and the beliefs of the players. Therefore, the analysis carried out in a finite or otherwise restrictive Harsanyi type space could be transferred, intact, to the universal Harsanyi type space where all the relevant states of affairs are present. The proof of the existence of a universal Harsanyi type space was extended to more general topological cases by Brandenburger and Dekel [3], Heifetz [9], Mertens et al. [14], and finally to the general measure theoretic case by Heifetz and Samet [12].

Knowledge (see, for example, [1,2] or [10]) is usually described by knowledge spaces. For each player, there is a partition of the underlying space of states of the world: For each state, the player knows an event if and only if the partition member that contains the state is contained in the event. Defined in this way, knowledge has the following properties: What is known by a player is true. A player knows what he knows and he knows what he does not know. Consequently, the assumptions on the epistemic attitudes of the players are rather strong in these models.

Recently, less demanding information structures have been considered in the literature in order to approach issues like bounded rationality or unawareness. Various standard solution concepts have been examined in these generalized information structures (see [4,7,17,18], and, for an overview, [5, Section 7] or [16, Chapter 3]). In such an information structure (or "Kripke structure", as it is called in Modal Logic (see [13])), each player's beliefs are described by a binary relation on the set of states of the world: For each state of the world, the player believes in an event if the event contains all those states of the world that are in relation with this state of the world. With each state of the world there is an associated state of nature ${ }^{1}$ which specifies the values of all the relevant objective parameters of the players' interaction. Examples of such objective parameters are the payoff functions, signals, or initial endowments. Imposing different restrictions on the relations of the players leads to different epistemic attitudes of the players (see [4-6], or [16] for more on this issue). For example, if the relations are equivalence relations, we are, in fact, in the case of knowledge spaces.

Heifetz and Samet [11] showed that, given at least two players and at least two states of nature, there is no universal knowledge space. However, as mentioned above, there is a universal Harsanyi type space.

One could imagine two potential sources for this discrepancy. Either, it is due to the different mathematical frameworks. That is, sets and binary relations (respectively partitions) on one side, and measurable spaces and probability measures on the other side. Or, alternatively, it is due to the strong assumptions on the players' epistemic attitudes in the case of knowledge, especially the truth axiom ("what a player knows in a state of the world is true in this state",

\footnotetext{
${ }^{1}$ When an information structure, respectively Kripke structure, is used as semantics for modal logic, a valuation function that describes which primitive propositions are true in a state of the world is used instead.
} 
corresponding on a technical level to reflexivity of the players' binary relations). These assumptions rule out the possibility that a player's type in some state of the world in a Harsanyi type space can be certain, with probability 1 , of some event which does not obtain in that state.

In the latter case, it could be possible that there is a universal information structure for classes of information structures with relaxed assumptions (in comparison to the case of knowledge) on the epistemic state of mind of the players. Since almost all classes of information structures considered in the literature contain the class of knowledge spaces, "relaxed" requires that knowledge spaces satisfy these assumptions as well.

To treat the question of the existence of a universal information structure rigorously, we define structure preserving maps between information structures, so called morphisms. Then, unfortunately, we show that we have to abandon the hope of having a universal information structure: Given at least two players and at least two states of nature, for every class of information structures that contains all knowledge spaces there is no universal information structure (Corollary 1). This is true even if we do not require the morphisms from the information structures to the universal information structure to be unique (Theorem 1).

The result is not proved by adapting the methods of the proof of Heifetz and Samet [11]. Instead, we use their result. The underlying idea is simple: Assuming by contradiction the existence of a universal information structure for a class of information structures that contains all knowledge spaces, we "collect" all those states lying in the images of knowledge spaces under morphisms, thus constructing a universal knowledge space, which, by the result of Heifetz and Samet, cannot exist.

\section{The nonexistence theorem}

In all what follows, let $S$ be a nonempty set of states of nature and $I$ a nonempty set of players.

Definition 1. An information structure on S for player set I ("information structure", for short) is a triplet

$$
\underline{M}:=\left\langle M,\left(\mathscr{K}_{i}\right)_{i \in I}, \theta\right\rangle
$$

where

- $M$ is a nonempty set,

- for $i \in I: \mathscr{K}_{i} \subseteq M \times M$,

- $\theta$ is function from $M$ to $S$.

$\theta$ relates the states of $M$, the so-called states of the world, to the states of nature. $\theta(m)$ is the state of nature that corresponds to the state of the world $m . \mathscr{K}_{i}$ is the possibility relation of player $i .(m, n) \in \mathscr{K}_{i}$ means that in the state $m$ player $i$ considers the state $n$ to be possible. 
Definition 2. An information structure $\underline{M}$ on $S$ for player set $I$ is called a knowledge space if for every $i \in I, \mathscr{K}_{i}$ is an equivalence relation.

The following definition captures the idea of mapping one information structure to another in a way that preserves the structure of the spaces.

Definition 3. Let $\underline{M}$ and $\underline{M}^{\prime}$ be information structures on $S$ for player set $I$. A morphism from $\underline{M}$ to $\underline{M}^{\prime}$ is a function $f: M \rightarrow M^{\prime}$ such that:

1. for all $m \in M$ :

$$
\theta^{\prime}(f(m))=\theta(m)
$$

2. for all $i \in I$ and $m \in M$ :

$$
f\left(\mathscr{K}_{i}(m)\right)=\mathscr{K}_{i}^{\prime}(f(m)),
$$

where

$$
\begin{aligned}
& \mathscr{K}_{i}(m):=\left\{n \in M \mid(m, n) \in \mathscr{K}_{i}\right\} \text { and } \\
& f(N):=\{f(n) \mid n \in N\}, \quad \text { for } N \subseteq M .
\end{aligned}
$$

Definition 4. Let $M$ be an information structure on $S$ for player set $I$. For an event $E \subseteq M$ define

$$
\begin{aligned}
& K_{i}(E):=\left\{m \in M \mid \mathscr{K}_{i}(m) \subseteq E\right\} . \\
& K_{i}: \operatorname{Pow}(M) \rightarrow \operatorname{Pow}(M)
\end{aligned}
$$

is called i's belief operator.

The following proposition, which is analogous to Proposition 2.1 of Heifetz and Samet [11], shows that Condition 2 of Definition 3 is equivalent to the preservation of beliefs in terms of the belief operators:

Proposition 1. Let $\underline{M}$ and $\underline{M}^{\prime}$ be information structures on $S$ for player set $I$. For a function $f: M \rightarrow M^{\prime}$ the following are equivalent:

1. For all $i \in I$ and $m \in M: f\left(\mathscr{K}_{i}(m)\right)=\mathscr{K}_{i}^{\prime}(f(m))$.

2. For all $i \in I$ and $E^{\prime} \subseteq M^{\prime}: f^{-1}\left(K_{i}^{\prime}\left(E^{\prime}\right)\right)=K_{i}\left(f^{-1}\left(E^{\prime}\right)\right)$.

This shows in particular that the morphisms defined by Heifetz and Samet [11], although defined for partition spaces, are the same as the ones defined here in the special case of knowledge spaces.

Proof. Note the following facts, which follow directly from the definitions:

$$
\begin{aligned}
m \in K_{i}\left(f^{-1}\left(E^{\prime}\right)\right) & \text { iff } \mathscr{K}_{i}(m) \subseteq f^{-1}\left(E^{\prime}\right) \\
& \text { iff } f\left(\mathscr{K}_{i}(m)\right) \subseteq E^{\prime},
\end{aligned}
$$


and

$$
\begin{aligned}
m \in f^{-1}\left(K_{i}^{\prime}\left(E^{\prime}\right)\right) & \text { iff } f(m) \in K_{i}^{\prime}\left(E^{\prime}\right) \\
& \text { iff } \mathscr{K}_{i}^{\prime}(f(m)) \subseteq E^{\prime} .
\end{aligned}
$$

We show first that 1. implies 2.: Let $i \in I$ and $E^{\prime} \subseteq M^{\prime}$. For all $m \in M$, we have $f\left(\mathscr{K}_{i}(m)\right)=\mathscr{K}_{i}^{\prime}(f(m))$ and hence for all $m \in M, f\left(\mathscr{K}_{i}(m)\right) \subseteq E^{\prime}$ iff $\mathscr{K}_{i}^{\prime}(f(m)) \subseteq E^{\prime}$. Therefore it follows for all $m \in M$ that $m \in K_{i}\left(f^{-1}\left(E^{\prime}\right)\right)$ iff $m \in f^{-1}\left(K_{i}^{\prime}\left(E^{\prime}\right)\right)$, that is $K_{i}\left(f^{-1}\left(E^{\prime}\right)\right)=f^{-1}\left(K_{i}^{\prime}\left(E^{\prime}\right)\right)$.

We show now that 2. implies 1.: Let $i \in I$ and $m \in M$. Letting $E^{\prime}=f\left(\mathscr{K}_{i}(m)\right)$ implies by 2 . that $\mathscr{K}_{i}^{\prime}(f(m)) \subseteq f\left(\mathscr{K}_{i}(m)\right)$, and letting $E^{\prime}=\mathscr{K}_{i}^{\prime}(f(m))$ implies by 2. that $f\left(\mathscr{K}_{i}(m)\right) \subseteq \mathscr{K}_{i}^{\prime}(f(m))$. Hence we have that $f\left(\mathscr{K}_{i}(m)\right)=\mathscr{K}_{i}^{\prime}(f(m))$.

Definition 5. Let $\mathscr{C}$ be a nonempty class of information structures on $S$ for player set I. An information structure on $S$ for player set $I$

$$
\underline{\Omega}^{\mathscr{C}}:=\left\langle\Omega,\left(\mathscr{K}_{i}^{\Omega}\right)_{i \in I}, \theta^{\Omega}\right\rangle
$$

is

- weak-universal for $\mathscr{C}$ if for every information structure $M \in \mathscr{C}$ there is a morphism from $\underline{M}$ to $\underline{\Omega}^{\mathscr{C}}$,

- universal for $\mathscr{C}$ if $\Omega^{\mathscr{C}} \in \mathscr{C}$ and for every information structure $\underline{M} \in \mathscr{C}$ there is a unique morphism from $\underline{M}$ to $\underline{\Omega}^{\mathscr{C}}$.

Theorem 1. Let $\mathscr{C}$ be a class of information structures on $S$ for player set $I$ that contains all knowledge spaces. If $S$ and I have each at least two elements, then there is no weak-universal information structure for $\mathscr{C}$.

Of course, a universal information structure for $\mathscr{C}$ is also a weak-universal information structure for $\mathscr{C}$, hence:

Corollary 1. Let $\mathscr{C}$ be a class of information structures on $S$ for player set $I$ that contains all knowledge spaces. If $S$ and I have each at least two elements, then there is no universal information structure for $\mathscr{C}$.

Let us now introduce some classes of information structures that have been used in the literature.

Definition 6. Let $M$ be a nonempty set. A binary relation $\mathscr{K}$ on $M$ is

- serial if for every $m \in M$ there is a $n \in M$ such that $(m, n) \in \mathscr{K}$,

- Euclidean if for all $m_{1}, m_{2}, m_{3} \in M:\left(m_{1}, m_{2}\right) \in \mathscr{K}$ and $\left(m_{1}, m_{3}\right) \in \mathscr{K}$ imply $\left(m_{2}, m_{3}\right) \in \mathscr{K}$.

Definition 7. Let $\mathscr{M}_{I}$ (respectively, $\left.\mathscr{M}_{I}^{\mathrm{r}} ; \mathscr{M}_{I}^{\mathrm{rt}} ; \mathscr{M}_{I}^{\mathrm{est}} ; \mathscr{P}_{\mathrm{I}}\right)$ be the class of all information structures on $S$ for player set $I$ (respectively the class of information structures on $S$ 
for player set $I$ where all the relations are reflexive; reflexive and transitive; Euclidean, serial and transitive; reflexive, symmetric and transitive (that is, knowledge spaces)).

These classes correspond to different axiom systems of modal logic, namely the systems $\mathrm{K}_{I}$ (respectively, $\mathrm{T}_{I} ; \mathrm{S} 4_{I} ; \mathrm{KD} 45_{I} ; \mathrm{S} 5_{I}$ ), see [6] for the definitions of these axiom systems. It is easy to see that all the above classes contain the class $\mathscr{P}_{I}$ (that is, the class of knowledge spaces).

Corollary 2. If $S$ and I each have at least two elements, then there is no weak-universal information structure for the class of all information structures on $S$ for player set I, the class $\mathscr{M}_{I}^{\mathrm{r}}$, the class $\mathscr{M}_{I}^{\mathrm{rt}}$, the class $\mathscr{M}_{I}^{\mathrm{est}}$, and the class $\mathscr{P}_{I}$.

Proof. As already remarked, every knowledge space on $S$ for player set $I$ belongs to each of these classes.

Corollary 3. If $S$ and I each have at least two elements, then there is no universal information structure for the class of all information structures on $S$ for player set $I$, the class $\mathscr{M}_{I}^{\mathrm{r}}$, the class $\mathscr{M}_{I}^{\text {rt }}$, the class $\mathscr{M}_{I}^{\mathrm{est}}$, and the class $\mathscr{P}_{I}$.

\section{The Proof}

Proof of Theorem 1. We use the nonexistence result of [11], where it was shown that for the class of knowledge spaces there is no weak-universal information structure which itself is a knowledge space. ${ }^{2}$ Now, let us assume on the contrary that there is a weak-universal information structure $\underline{\Omega}^{\mathscr{C}}=\left\langle\Omega,\left(\mathscr{K}_{i}^{\Omega}\right)_{i \in I}, \theta^{\Omega}\right\rangle$ for $\mathscr{C}$. According to the assumptions of the theorem, $\mathscr{P}_{I}$ is contained in $\mathscr{C}$. Let

$$
\Omega^{\mathscr{P}}:=\left\{\omega \in \Omega \mid \exists \underline{M} \in \mathscr{P}_{I} \exists \text { morphism } h: \underline{M} \rightarrow \underline{\Omega}^{\mathscr{C}} \text { such that } \omega \in h(M)\right\} .
$$

Now define

$$
\underline{\Omega}^{\mathscr{P}}:=\left\langle\Omega^{\mathscr{P}},\left(\mathscr{K}_{i}^{\Omega} \cap\left(\Omega^{\mathscr{P}} \times \Omega^{\mathscr{P}}\right)\right)_{i \in I}, \theta^{\Omega}\left\lceil\Omega^{\mathscr{P}}\right\rangle .\right.
$$

We have to show:

1. $\Omega^{\mathscr{P}}$ is a knowledge space,

2. $\Omega^{\mathscr{P}}$ is weak-universal for $\mathscr{P}_{I}$.

This implies the desired contradiction.

1. $\Omega^{\mathscr{P}}$ is nonempty, because $\mathscr{P}_{I}$ is nonempty. That $\theta\left\lceil\Omega^{\mathscr{P}}\right.$ is a function from $\Omega^{\mathscr{P}}$ to $S$ is clear. Fix a player $i \in I$.

\footnotetext{
${ }^{2}$ Heifetz and Samet [11] state just that there is no universal knowledge space. However, in that paper their use of "universal" is identical with our use of "weak-universal" here.
} 
Let $\omega_{1} \in \Omega^{\mathscr{P}}$. Then, there is a knowledge space $\underline{M}$ in $\mathscr{P}_{I}, m_{1} \in M$, and a morphism $h$ from $M$ to $\Omega^{\mathscr{C}}$ such that $h\left(m_{1}\right)=\omega_{1}$. But then, $\left(m_{1}, m_{1}\right) \in \mathscr{K}_{i}^{M}$, hence $\left(h\left(m_{1}\right), h\left(m_{1}\right)\right) \in \mathscr{K}_{i}^{\Omega}$, so $\left(h\left(m_{1}\right), h\left(m_{1}\right)\right) \in \mathscr{K}_{i}^{\Omega} \cap\left(\Omega^{\mathscr{P}} \times \Omega^{\mathscr{P}}\right)$, that is, $\mathscr{K}_{i}^{\Omega} \cap\left(\Omega^{\mathscr{P}} \times\right.$ $\left.\Omega^{\mathscr{P}}\right)$ is reflexive.

Let $\omega_{1}, \omega_{2} \in \Omega^{\mathscr{P}}$ and $\left(\omega_{1}, \omega_{2}\right) \in \mathscr{K}_{i}^{\Omega} \cap\left(\Omega^{\mathscr{P}} \times \Omega^{\mathscr{P}}\right)$. Then, there is a knowledge space $\underline{M}$ in $\mathscr{P}_{I}, m_{1} \in M$, and a morphism $h$ from $\underline{M}$ to $\underline{\Omega}^{\mathscr{C}}$ such that $h\left(m_{1}\right)=\omega_{1}$. We have $\omega_{2} \in \mathscr{K}_{i}^{\Omega}\left(h\left(m_{1}\right)\right)=h\left(\mathscr{K}_{i}^{M}\left(m_{1}\right)\right)$. So there is a $m_{2} \in M$ such that $\omega_{2}=$ $h\left(m_{2}\right)$ and $\left(m_{1}, m_{2}\right) \in \mathscr{K}_{i}^{M}$. But then $\left(m_{2}, m_{1}\right) \in \mathscr{K}_{i}^{M}$ and therefore $\left(\omega_{2}, \omega_{1}\right) \in \mathscr{K}_{i}^{\Omega} \cap\left(\Omega^{\mathscr{P}} \times \Omega^{\mathscr{P}}\right)$, so $\mathscr{K}_{i}^{\Omega} \cap\left(\Omega^{\mathscr{P}} \times \Omega^{\mathscr{P}}\right)$ is symmetric.

Let $\omega_{1}, \omega_{2}, \omega_{3} \in \Omega^{\mathscr{P}}$ and $\left(\omega_{1}, \omega_{2}\right),\left(\omega_{2}, \omega_{3}\right) \in \mathscr{K}_{i}^{\Omega} \cap\left(\Omega^{\mathscr{P}} \times \Omega^{\mathscr{P}}\right)$. Then, as above, there is a knowledge space $\underline{M}$ in $\mathscr{P}_{I}, m_{1}, m_{2} \in M$, and a morphism $h$ from $\underline{M}$ to $\underline{\Omega}^{\mathscr{C}}$ such that $\left(m_{1}, m_{2}\right) \in \mathscr{K}_{i}^{M}, \quad h\left(m_{1}\right)=\omega_{1}, \quad$ and $h\left(m_{2}\right)=\omega_{2}$. We have $\omega_{3} \in \mathscr{K}_{i}^{\Omega}\left(h\left(m_{2}\right)\right)=h\left(\mathscr{K}_{i}^{M}\left(m_{2}\right)\right)$. So there is a $m_{3} \in M$ such that $\omega_{3}=h\left(m_{3}\right)$ and $\left(m_{2}, m_{3}\right) \in \mathscr{K}_{i}^{M}$. Then, $\left(m_{1}, m_{3}\right) \in \mathscr{K}_{i}^{M}$, so it follows that $\omega_{3} \in h\left(\mathscr{K}_{i}^{M}\left(m_{1}\right)\right)=$ $\mathscr{K}_{i}^{\Omega}\left(\omega_{1}\right)$. Therefore $\left(\omega_{1}, \omega_{3}\right) \in \mathscr{K}_{i}^{\Omega} \cap\left(\Omega^{\mathscr{P}} \times \Omega^{\mathscr{P}}\right)$, so $\mathscr{K}_{i}^{\Omega} \cap\left(\Omega^{\mathscr{P}} \times \Omega^{\mathscr{P}}\right)$ is transitive.

2. Let $\underline{M} \in \mathscr{P}_{I}$. Then there is a morphism $h$ from $\underline{M}$ to $\underline{\Omega}^{\mathscr{C}}$. For $m \in M$ is $h(m) \in \Omega^{\mathscr{P}}$ and

$$
\theta^{\Omega}\left\lceil\Omega^{\mathscr{P}}(h(m))=\theta^{\Omega}(h(m))=\theta^{M}(m) .\right.
$$

We have $h\left(\mathscr{K}_{i}^{M}(m)\right) \subseteq \Omega^{\mathscr{P}}$ and $h(m) \in \Omega^{\mathscr{P}}$, so

$$
h\left(\mathscr{K}_{i}^{M}(m)\right)=\mathscr{K}_{i}^{\Omega}(h(m))=\mathscr{K}_{i}^{\Omega} \cap\left(\Omega^{\mathscr{P}} \times \Omega^{\mathscr{P}}\right)(h(m)) .
$$

Hence $h$ is a morphism from $\underline{M}$ to $\underline{\Omega}^{\mathscr{P}}$.

\section{Acknowledgments}

I am greatly indebted to Dov Samet and an anonymous referee for very helpful comments that clarified and improved the presentation of the paper a lot. Helpful remarks of Enrico Minelli, Aviad Heifetz, Vincent Merlin, Hamish Waterer, and Antoinette Baujard are gratefully acknowledged. Most of this work was done when the author was a TMR-fellow of the TMR network "Cooperation and Information", ERB FMRX CT 0055, at the Université de Caen, I am grateful for this support and thank Maurice Salles, Vincent Merlin, and the Université de Caen for their hospitality. I am the only person responsible for remaining errors.

\section{References}

[1] R.J. Aumann, Agreeing to disagree, Ann. Statist. 4 (1976) 1236-1239.

[2] R.J. Aumann, Interactive epistemology I: knowledge, Int. J. Game Theory 28 (1999) 263-300. 
[3] A. Brandenburger, E. Dekel, Hierarchies of beliefs and common knowledge, J. Econ. Theory 59 (1993) 189-198.

[4] A. Brandenburger, E. Dekel, J. Geanakoplos, Correlated equilibrium with generalized information structures, Games Econ. Behav. 4 (1992) 182-201.

[5] E. Dekel, F. Gul, Rationality and knowledge in game theory, in: D.M. Kreps, K.F. Wallis (Eds.), Advances in Economics and Econometrics: Theory and Applications, Cambridge University Press, Cambridge, UK, 1997.

[6] R. Fagin, J.Y. Halpern, Y. Moses, M. Vardi, Reasoning about knowledge, MIT Press, Cambridge, MA, 1995.

[7] J. Geanakoplos, Game theory without partitions, and applications to speculation and consensus, Mimeo, Cowles Foundation, Yale University, 1989.

[8] J.C. Harsanyi, Games with incomplete information played by Bayesian players, parts I, II, III, Manage. Sci. 14 (1967-1968) 159-182, 320-334, 486-502.

[9] A. Heifetz, The Bayesian formulation of incomplete information-the non-compact case, Int. J. Game Theory 21 (1993) 329-338.

[10] A. Heifetz, Infinitary S5-epistemic logic, Math. Logic Quart. 43 (1997) 333-342.

[11] A. Heifetz, D. Samet, Knowledge spaces with arbitrarily high rank, Games Econ. Behav. 22 (1998) 260-273.

[12] A. Heifetz, D. Samet, Topology-free typology of beliefs, J. Econ. Theory 82 (1998) 324-341.

[13] S.A. Kripke, Semantical analysis of modal logic I. Normal modal propositional calculi, Z. fuer Math. Logik Grundlagen Math. 9 (1963) 363-374.

[14] J.F. Mertens, S. Sorin, S. Zamir, Repeated games. A. Background material, CORE Discussion Paper 9420, 1994.

[15] J.F. Mertens, S. Zamir, Formulation of Bayesian analysis for games with incomplete information, Int. J. Game Theory 14 (1985) 1-29.

[16] A. Rubinstein, Modeling Bounded Rationality, MIT Press, Cambridge, MA, 1998.

[17] A. Rubinstein, A. Wolinsky, On the logic of 'agreeing to disagree' type results, J. Econ. Theory 51 (1989) 184-193.

[18] D. Samet, Ignoring ignorance and agreeing to disagree, J. Econ. Theory 52 (1990) 190-207. 